\title{
Correction to: Correlation analysis on serum inflammatory cytokine level and neurogenic pulmonary edema for children with severe hand-foot-mouth disease
}

\author{
Jin-Fang Sun, Hao-Lan Li ${ }^{*}$ and Bao-Xia Sun
}

\section{Correction to: Eur J Med Res (2018) 23:21.} https://doi.org/10.1186/s40001-018-0313-1

The original publication of this article [1] contained two erroneous paragraphs related to the time and place for the admission of the pediatric patients with clinically diagnosed severe HFMD. The updated information has been indicated in bold.

- Incorrect: In the present study, the dynamic changes in inflammatory cytokine levels in patients with severe HFMD after admission, who were treated and cured in two hospitals in Henan Province, China from March 2010 to December 2012, were monitored.

- Correct: In the present study, the dynamic changes in inflammatory cytokine levels in patients with severe HFMD after admission, who were treated and cured in two hospitals in Shandong Province, China from March 2012 to December 2016, were monitored.

- Incorrect: Case selection: pediatric patients with clinically diagnosed severe HFMD, who were admitted at Zhengzhou Children's Hospital and Henan Infectious Hospital from March 2010 to December 2012, were included into the present study.
- Correct: Case selection: pediatric patients with clinically diagnosed severe HFMD, who were admitted at our hospital from March 2012 to December 2016, were included into the present study.

The original article can be found online at https://doi.org/10.1186/ s40001-018-0313-1.

\section{Publisher's Note}

Springer Nature remains neutral with regard to jurisdictional claims in published maps and institutional affiliations.

Received: 16 May 2018 Accepted: 18 May 2018 Published online: 15 June 2018

\section{Reference}

1. Sun JF, Li HL, Sun BX. Correlation analysis on serum inflammatory cytokine level and neurogenic pulmonary edema for children with severe hand-foot-mouth disease. Eur J Med Res. 2018;23:21. https://doi. org/10.1186/s40001-018-0313-1. 